\title{
Inhibition of mechanosensitive signaling in myofibroblasts ameliorates experimental pulmonary fibrosis
}

\author{
Yong Zhou, Xiangwei Huang, Louise Hecker, Deepali Kurundkar, Ashish Kurundkar, Hui Liu, \\ Tong-Huan Jin, Leena Desai, Karen Bernard, and Victor J. Thannickal
}

Division of Pulmonary, Allergy and Critical Care Medicine, Department of Medicine, University of Alabama at Birmingham, Birmingham, Alabama, USA.

\begin{abstract}
Matrix stiffening and myofibroblast resistance to apoptosis are cardinal features of chronic fibrotic diseases involving diverse organ systems. The interactions between altered tissue biomechanics and cellular signaling that sustain progressive fibrosis are not well defined. In this study, we used ex vivo and in vivo approaches to define a mechanotransduction pathway involving Rho/Rho kinase (Rho/ROCK), actin cytoskeletal remodeling, and a mechanosensitive transcription factor, megakaryoblastic leukemia 1 (MKL1), that coordinately regulate myofibroblast differentiation and survival. Both in an experimental mouse model of lung fibrosis and in human subjects with idiopathic pulmonary fibrosis (IPF), we observed activation of the Rho/ROCK pathway, enhanced actin cytoskeletal polymerization, and MKL1 cytoplasmic-nuclear shuttling. Pharmacologic disruption of this mechanotransduction pathway with the ROCK inhibitor fasudil induced myofibroblast apoptosis through a mechanism involving downregulation of BCL-2 and activation of the intrinsic mitochondrial apoptotic pathway. Treatment with fasudil during the postinflammatory fibrotic phase of lung injury or genetic ablation of $M k l 1$ protected mice from experimental lung fibrosis. These studies indicate that targeting mechanosensitive signaling in myofibroblasts to trigger the intrinsic apoptosis pathway may be an effective approach for treatment of fibrotic disorders.
\end{abstract}

\section{Introduction}

Fibrotic diseases encompass a diverse group of disorders, of known and unknown etiologies, that affect multiple organ systems and contribute to significant morbidity and mortality (1-3). Myofibroblasts are key effector cells in fibrotic disorders characterized by persistent or progressive fibrosis (1). Myofibroblasts also participate in normal wound healing by facilitating wound closure and synthesis of ECM proteins (4). Termination of the reparative response that leads to normal wound healing is heralded by the apparent disappearance of myofibroblasts from granulation tissue $(1,5,6)$. This disappearance may involve dedifferentiation of myofibroblasts to the quiescent progenitor phenotype (7) or clearance of apoptotic or senescent myofibroblasts (8-10). In contrast, the persistence of myofibroblasts in injured tissues leads to nonresolving and progressive fibrosis, as exemplified by human idiopathic pulmonary fibrosis (IPF) (11).

Myofibroblasts acquire contractile activity that is similar - but not identical - to that of smooth muscle cells $(12,13)$. Enhanced contractility of myofibroblasts is an important phenotypic characteristic of myofibroblast differentiation $(5,14,15)$. However, it remains largely unknown whether myofibroblast contractility can reciprocally regulate its differentiation and fate. Recent studies suggest that myofibroblast contraction provides a feed-forward mechanism for maintaining myofibroblastic phenotype through the conversion of mechanical stimuli into fibrogenic signals, primarily via the extrinsic mechanotransduction involving activation of latent TGF- $\beta$ (16-19). In contrast, we recently identified an intrinsic mechanotransduction pathway in which megakaryoblastic leukemia 1 (MKL1) converts mechanical stimuli derived from

Conflict of interest: The authors have declared that no conflict of interest exists. Citation for this article: J Clin Invest. 2013;123(3):1096-1108. doi:10.1172/JCI66700. lung fibroblast contraction in response to matrix stiffening into a fibrogenic nuclear signal that promotes fibroblast-to-myofibroblast differentiation (20). In this study, we investigated the role of myofibroblast contractility in the regulation of myofibroblast differentiation and fate in response to both biochemical (i.e., TGF$\beta 1$ ) and biomechanical (i.e., matrix stiffening) stimuli.

The Rho kinase (ROCK) family members, consisting of ROCK1 (also known as ROK $\beta$ or p160ROCK) and ROCK2 (also known as $\mathrm{ROK} \alpha$ ), are serine/threonine kinases that are activated by Rho GTPases. ROCKs regulate important cellular functions, including proliferation, migration, adhesion, and apoptosis/survival (21). ROCK-mediated effects are elicited by phosphorylation of downstream targets, many of which are associated with the regulation of cell contractility, actin cytoskeletal organization, stress fiber formation, and focal adhesion assembly (22). Mice deficient in ROCK1 are protected from cardiac fibrosis in response to pressure overload $(23,24)$. This protective effect is likely the result of impaired sensing and/or responses of cardiac fibroblasts to biomechanical stress. ROCK inhibitors are in development for the treatment of a number of cardiovascular disorders (25). In a lung injury model using intratracheal bleomycin, simultaneous administration of the ROCK inhibitor Y-27632 inhibited neutrophil and macrophage infiltration as well as fibroblast proliferation and migration (26). However, this experimental design limits interpretation of the potential efficacy of the antifibrotic (versus antiinflammatory) effects of ROCK inhibition; importantly, the role of targeting the ROCK pathway to modulate biomechanical signaling of myofibroblast differentiation and fate are uncertain.

Fasudil is a small-molecule inhibitor of ROCK that has been approved for the treatment of cerebral vasospasm in Japan (27). Fasudil is composed of 2 functional groups, an isoquinoline ring and a homopiperazine ring, connected by a sulfonamide linker 
(28). It inhibits ROCK by competing with ATP binding at the hydrophobic cleft between $\mathrm{N}$ - and C-terminal lobes of the ROCK kinase domain (29). Specifically, the homopiperazine ring attaches the entrance of the cleft where active residues are clustered, while the planar isoquinoline ring inserts into the canonical adenine binding pocket (29).

In this study, we aimed to establish proof-of-concept that targeting mechanosensitive signaling pathways that regulate myofibroblast differentiation and promote myofibroblast survival may serve as an effective antifibrotic therapeutic strategy. We tested the effects of fasudil in the modulation of myofibroblast differentiation and survival in vivo and in vitro. Our findings strongly support a role for a mechanotransduction pathway involving the Rho/ ROCK pathway, enhanced actin cytoskeletal polymerization, and MKL1 in sustained myofibroblast activation. The present study also uncovered a novel MKL1 transcriptional target (BCL-2) that promotes myofibroblast survival.

\section{Results}

Fasudil induces lung myofibroblast apoptosis in vitro and in vivo, while normal lung fibroblasts are not susceptible to fasudil-induced cell death. Myofibroblast apoptosis heralds the termination of the reparative response to tissue injury (8), and resistance to apoptosis of myofibroblasts has been associated with persistence of fibrosis (30). To determine the effects of fasudil, a ROCK inhibitor, on apoptosis of lung myofibroblasts and normal lung fibroblasts in vitro, we isolated (myo)fibroblasts from lungs of patients with IPF and from failed donor lungs. Myofibroblasts were identified by expression of $\alpha$-SMA (Figure 1A). Fasudil treatment significantly increased the number of apoptotic cells in $\alpha$-SMA-positive lung myofibroblasts at 24 and 48 hours, whereas normal lung fibroblasts were insensitive to the apoptosis-inducing effects of fasudil (Figure 1, A and B). To determine the effects of fasudil on apoptosis of myofibroblasts differentiated from TGF- $\beta 1$-treated normal lung fibroblasts, we cultured normal lung fibroblasts in the presence of TGF- $\beta 1$. Fibroblast-to-myofibroblast differentiation was evident by the formation of $\alpha$-SMA-positive stress fibers (Figure 1A). Similar to IPF myofibroblasts, fasudil treatment induced a majority of myofibroblasts derived from TGF$\beta 1$-treated normal fibroblasts to undergo apoptosis (Figure 1, $\mathrm{A}$ and $\mathrm{B})$. To determine whether fasudil induces myofibroblast apoptosis in vivo, we injured lungs of mice with intratracheal instillation of the chemotherapeutic agent bleomycin. Control mice were administered saline. On days 14-27 after bleomycin injury (i.e., the postinflammatory fibrotic phase), mice were administered daily intraperitoneal injections of $25 \mathrm{mg} / \mathrm{kg}$ fasudil or PBS as a control. Mouse lungs were harvested on day 28. In situ TUNEL and costaining with $\alpha$-SMA showed that in bleomycin-injured mice receiving PBS, cellular apoptosis was primarily localized to the alveolar lining epithelium, whereas interstitial $\alpha$-SMA-positive lung cells in fibrotic regions were largely apoptosis resistant (Figure 1, C and D). In contrast, fasudil treatment of injured mice resulted in the appearance of TUNEL-positive, $\alpha$-SMA-expressing cells within alveolar walls (Figure 1, C and E). Smooth muscle cells in bronchioles and pulmonary arteries did not undergo significant apoptosis in response to fasudil (Supplemental Figure 1; supplemental material available online with this article; doi:10.1172/JCI66700DS1). These data suggest that fasudil treatment selectively promotes myofibroblasts to undergo apoptosis ex vivo and in vivo.
Fasudil induces lung myofibroblast apoptosis by downregulation of BCL-2 expression. The ROCK pathway regulates actin dynamics, which may influence susceptibility to apoptosis via the mitochondrial pathway (21). The release of cytochrome $c$ from mitochondria is an essential step for activation of the intrinsic apoptosis pathway (31). We first examined whether fasudil induces the activation of mitochondrial cytochrome $c$ release. Treatment of IPF (myo)fibroblasts with fasudil induced a time-dependent release of cytochrome $c$ from mitochondria (Figure 2A). A decrease in the level of cytochrome $c$ in mitochondria fraction was observed 8-24 hours after fasudil treatment; concordantly, the level of cytochrome $c$ in the cytosolic fraction increased during the same time period.

B cell lymphoma 2 (BCL-2), the prototypic member of the BCL-2 family that controls mitochondrial outer membrane permeabilization, is known to inhibit the release of cytochrome $c$ from mitochondria (32). We next sought to determine whether fasudil regulates BCL-2 expression in lung myofibroblasts. Whereas fasudil downregulated mRNA and protein expression of BCL-2 in a dosedependent manner in IPF lung myofibroblasts at 24 hours, mRNA and protein levels of Bcl-xL and Mlc-1, 2 additional members of the BCL-2 family, were not influenced by fasudil treatment (Figure 2, B and C). Furthermore, fasudil-induced downregulation of BCL-2 expression was associated with activation of caspase 9 and caspase 3 , as evidenced by proenzyme cleavage and increased enzymatic activities (Figure 2D). Fasudil did not activate caspase 8, a downstream effector of the death receptor-mediated extrinsic apoptotic pathway (Supplemental Figure 2). Collectively, these results indicate that fasudil mediates downregulation of BCL-2 expression and activates the intrinsic mitochondrial apoptotic pathway.

In contrast to the proapoptotic effects of fasudil on IPF myofibroblasts, this ROCK inhibitor did not sensitize control fibroblasts to activation of the mitochondrial apoptotic pathway, as fasudil treatment did not induce cytochrome $c$ release, regulate BCL-2 protein expression, or activate caspase 9/3 (Supplemental Figure 3). Thus, fasudil selectively induced myofibroblast apoptosis by downregulating BCL-2 expression and activating the intrinsic mitochondrial apoptosis pathway. To determine whether this prosurvival BCL-2 pathway is constitutively upregulated in human IPF myofibroblasts, we performed immunoblot analyses of cell lysates from non-IPF control fibroblasts and IPF myofibroblasts, the latter of which expressed significantly higher levels of BCL-2 (Figure 2E).

Fasudil downregulates $B C L-2$ expression by deactivation of MKL1mediated intrinsic mechanotransduction. MKL1 is an actin dynamics sensor and SRF coactivator that plays a critical role in the activation of a number of profibrotic genes (20,33-38). When G-actin is polymerized into F-actin, MKL1 is released from G-actin and enters into the nucleus, where it binds to SRF and targets CArG consensus sequence(s) in the promoter region to activate gene transcription (38). A bioinformatics search revealed 2 CArG boxes, box1 (approximately -565 to $-558 \mathrm{bp}$ ) and box2 (approximately $-1,190$ to $-1,181 \mathrm{bp}$ ), in the proximal control region of the human $B C L 2$ gene. To determine whether fasudil-regulated $B C L 2$ gene expression in lung myofibroblasts is mediated by MKL1, we compared MKL1 cytoplasmic-nuclear shuttling in IPF lung myofibroblasts and control lung fibroblasts. MKL1 subcellular localization analysis showed that IPF lung myofibroblasts expressed higher levels of MKL1 in the nuclear fraction than did control cells (Figure 3A). G-actin and F-actin content analysis demonstrated that lung myofibroblasts contained more F-actin in the total actin pool 
A
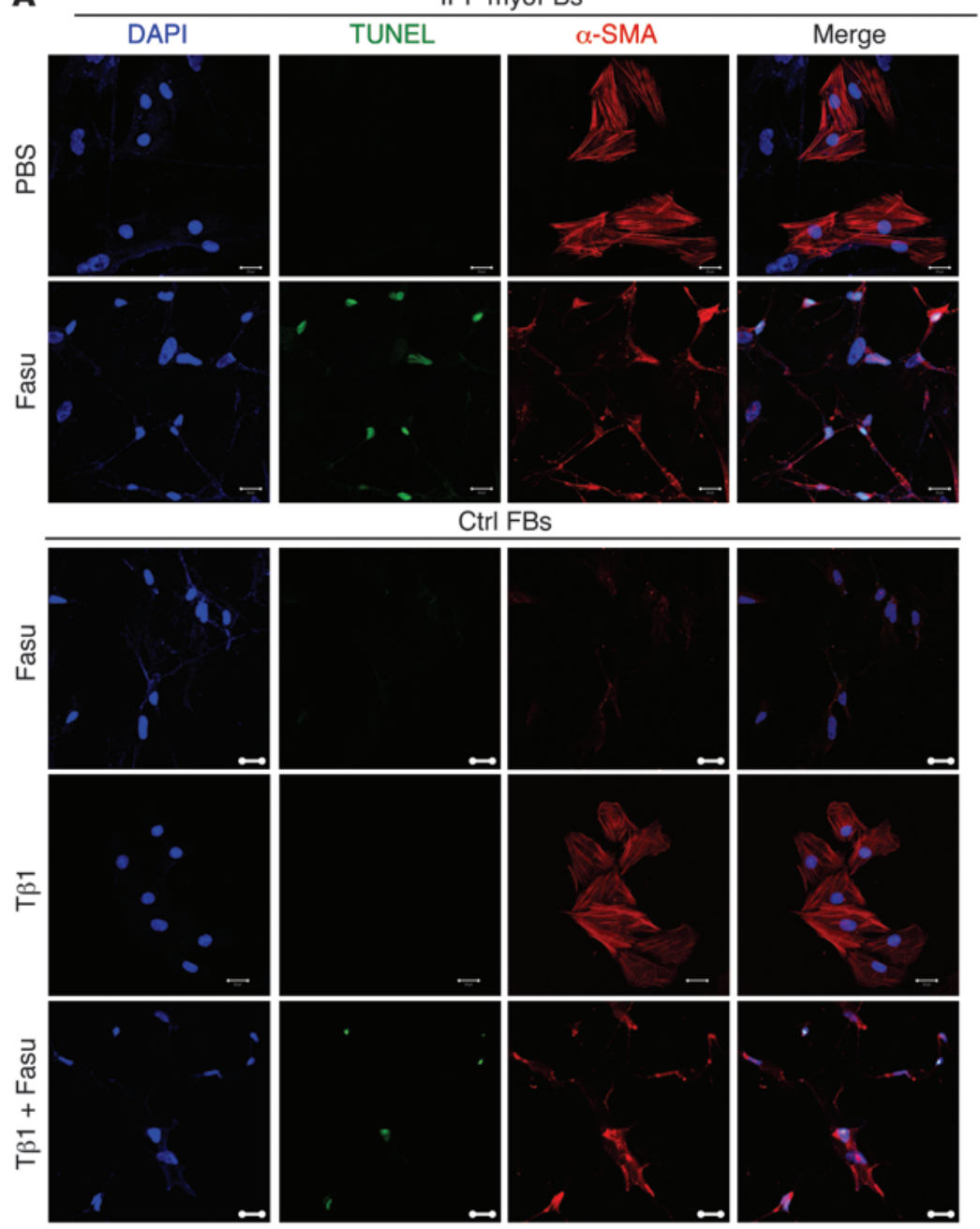

C TUNEL + DAPI
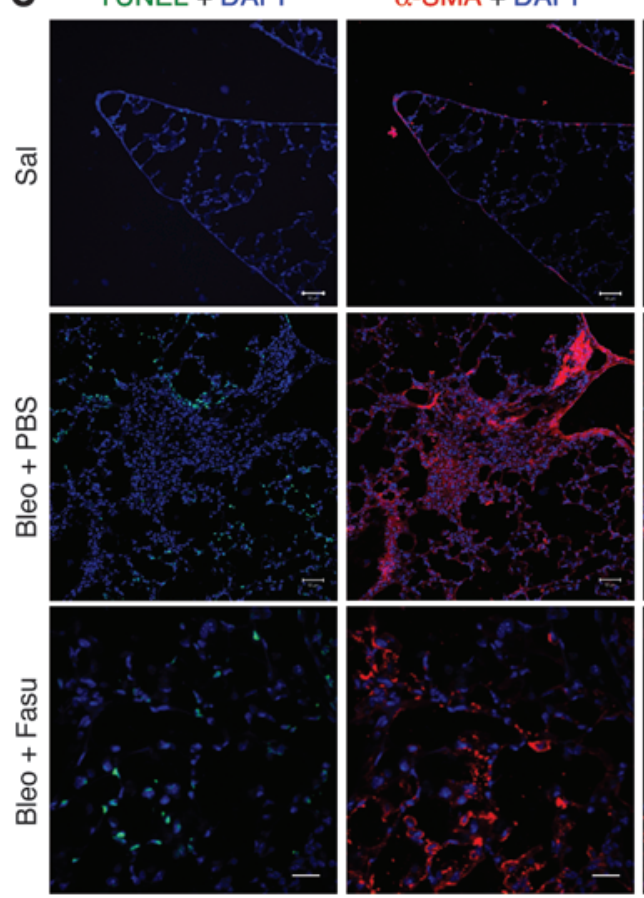

$\alpha-S M A+D A P I$
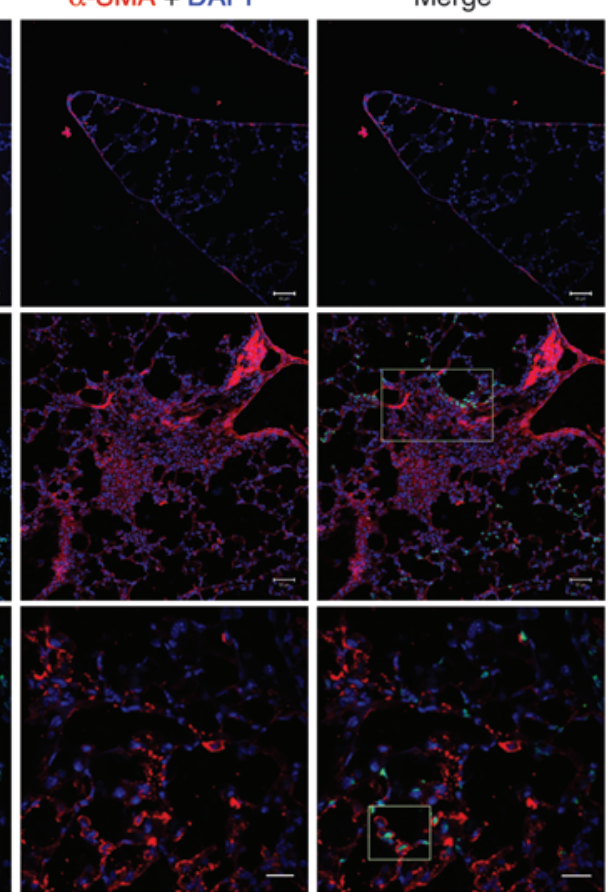

B

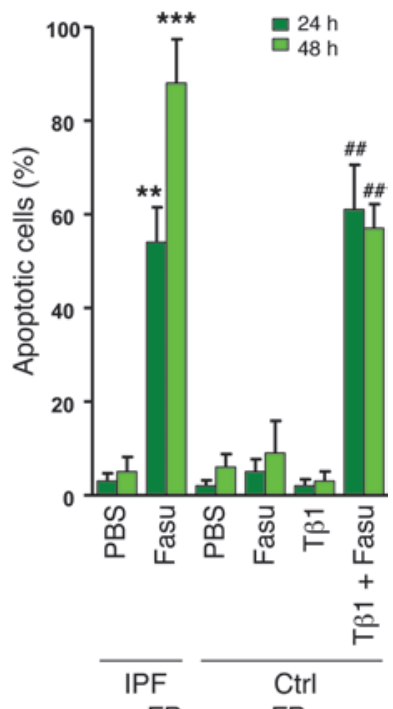

myoFBs FBs 


\section{Figure 1}

Fasudil induces myofibroblast apoptosis both in vitro and in vivo, while normal lung fibroblasts are not susceptible to fasudil-induced cell death. (A) Myofibroblasts (myoFBs) isolated from lungs of patients with IPF $(n=8)$ and fibroblasts of non-IPF control subjects (failed donors; $n=6$ ) were cultured in the presence or absence of $25 \mu \mathrm{M}$ fasudil (Fasu) for 24-48 hours. Control fibroblasts (Ctrl FBs) were treated with $4 \mathrm{ng} / \mathrm{ml} \mathrm{TGF-} \beta 1$ (T $\beta 1$ ) and/or fasudil. Cells were double stained for TUNEL (green) and $\alpha$-SMA (red); nuclei were stained with DAPI (blue). Confocal fluorescent images were overlaid to show apoptotic myofibroblasts. Scale bars: $20 \mu \mathrm{M}$. (B) Apoptotic cells (i.e., TUNEL positive) were quantified and expressed as the percentage of total cells. At least 300 cells were counted per condition. ${ }^{\star \star} P<0.01$, ${ }^{* * *} P<0.001$ vs. PBS; ${ }^{\# \# P}<0.01$ vs. TGF- $\beta 1$. (C-E) Frozen lung tissue sections from mice treated with saline (Sal) or with bleomycin (Bleo) in combination with PBS or fasudil were stained for TUNEL, $\alpha-S M A$, and nuclei as in A. Higher-magnification views of the boxed regions in the bleomycin plus PBS and bleomycin plus fasudil images are shown in $\mathbf{D}$ and $\mathbf{E}$, respectively (enlarged $\times 4-$ to $\times 5$-fold). Scale bars: $50 \mu \mathrm{M}$.

than control lung fibroblasts (Figure 3B). Levels of total actin were found to be equivalent in lung myofibroblasts and control lung fibroblasts (Supplemental Figure 4). These data indicate that increased F-actin polymerization is linked to the constitutive activation of MKL1 nuclear signaling in IPF lung myofibroblasts.

In IPF lung myofibroblasts, fasudil treatment decreased MKL1 levels in the nuclear fraction while increasing MKL1 expression in the cytoplasm (Figure 3C), which suggests that fasudil "deactivates" constitutive MKL1 nuclear signaling in "diseased" cells. No apparent changes in MKL1 subcellular localization were observed in control lung fibroblasts in response to fasudil treatment. Whereas fasudil markedly reduced F-actin content in lung myofibroblasts, only a slight decrease in F-actin content was observed in normal lung fibroblasts in response to fasudil treatment (Figure 3D). These data indicate that fasudil inhibits F-actin polymerization and deactivates MKL1 signal activation in lung myofibroblasts.

To further determine whether fasudil deactivation of MKL1 nuclear signaling is responsible for the observed downregulation of $B C L 2$ gene expression, we performed quantitative ChIP assays to examine the effect of fasudil on the binding of MKL1-SRF complex to the BCL2 promoter. The constitutive enrichment of BCL2 promoter DNA in SRF antibody-immunoprecipitated chromatin of IPF myofibroblasts was suppressed by fasudil (Figure 3E).

Next, we determined whether the fasudil-induced decrease in the binding of MKL1-SRF to the BCL2 promoter inhibits BCL2 promoter activity. A 1,096-bp WT human proximal BCL2 promoter reporter and 3 mutated promoter reporters harboring mutations at the specific MKL1-SRF-binding DNA sequences - CArG box1, CArG box2, or both box1 and box 2 - were transfected into lung myofibroblasts (Figure $3 \mathrm{~F}$ ). In cells transfected with the WT $B C L 2$ promoter reporter, fasudil significantly decreased luciferase expression (Figure $3 \mathrm{~F}$ ), which suggests that fasudil inhibits BCL2 promoter activity. Mutation at CArG box1 neither decreased constitutive $B C L 2$ promoter activity nor abrogated fasudil inhibition of this activity (Figure 3F), which suggests that it is not involved in BCL-2 regulation. Mutation of CArG box 2 and of box 1 and box 2 combined diminished baseline $B C L 2$ promoter activities in lung myofibroblasts (Figure 3F), which indicates that constitutive promoter activity is MKL1-SRF dependent. Collectively, these results suggest that CArG box2 (approximately $-1,190$ to -1,181 bp of the
$B C L 2$ promoter) is responsible for MKL1-dependent constitutive activation of $B C L 2$ gene expression in lung myofibroblasts. These data indicate that fasudil downregulates $B C L 2$ gene expression by inhibiting MKL1-SRF complex binding to CArG box2.

Forced nuclear translocation of MKL1 by treatment with jasplakinolide, a stabilizer of F-actin (38), or by overexpression of constitutively active (i.e., nuclear) MKL1 (33) rescued fasudil downregulation of BCL-2 expression in lung myofibroblasts (Figure $3 \mathrm{G}$ ). In contrast, inhibition of MKL1 nuclear signaling by disruption of F-actin polymerization with latrunculin B (39), blockade of MKL1-SRF complex binding to the BCL2 promoter with CCG-1423 (40), or overexpression of dominant-negative MKL1 (dnMKL1) (33) downregulated BCL-2 expression in lung myofibroblasts (Figure $3 \mathrm{H}$ ), effects that were equivalent to those with fasudil treatment. Taken together, these data indicate that fasudil downregulation of BCL-2 expression in lung myofibroblasts is mediated, at least in part, by deactivation of actin cytoskeletondependent MKL1 nuclear signaling.

Fasudil prevents lung fibroblast-to-myofibroblast differentiation in response to TGF- $\beta 1$ and matrix stiffness. Fibroblast-to-myofibroblast differentiation is characterized by $\alpha$-SMA expression and enhanced contractile activity (4). While fibrogenic cytokines/growth factors are well established as promoters of fibroblast-to-myofibroblast differentiation, it has only recently been appreciated that the mechanical properties of ECM (i.e., matrix stiffness) serve as an important regulator of myofibroblast differentiation $(20,41)$. We therefore sought to determine whether fasudil blocks lung fibroblast-to-myofibroblast differentiation in response to TGF- $\beta 1$ and matrix stiffness. Fibroblast-to-myofibroblast differentiation was induced by TGF- $\beta 1$ and/or matrix stiffening in the presence or absence of fasudil. Quantitative real-time PCR and immunoblot analyses showed that TGF- $\beta 1$ and matrix stiffening alone or in combination promoted significant increases in mRNA and protein expression of $\alpha$-SMA, indicative of myofibroblast differentiation, whereas fasudil abrogated the induction of $\alpha$-SMA protein and mRNA expression under these conditions (Figure 4, A and B). Confocal immunofluorescent microscopy showed that TGF- $\beta 1$ and/or matrix stiffening promoted $\alpha$-SMA incorporation into stress fibers; cotreatment with fasudil blocked $\alpha$-SMA-positive stress fiber formation in response to both TGF- $\beta 1$ and matrix stiffening (Figure $4 \mathrm{C}$ ). These data suggest that fasudil is capable of blocking the integrated signaling from biochemical (TGF- $\beta 1$ ) and biomechanical (matrix stiffness) cues that mediate myofibroblast differentiation.

(Myo)fibroblast contractility is associated with the phosphorylation of myosin light chain $\left(\mathrm{MLC}_{20}\right)$ (4). We detected the highest levels of $\mathrm{MLC}_{20}$ phosphorylation in fibroblasts grown on stiff matrix treated with TGF- $\beta 1$, followed by fibroblasts grown on stiff matrix in the absence of treatment, then fibroblasts grown on soft matrix treated with TGF- $\beta 1$; fasudil treatment inhibited these responses to both soluble and matrix signals (Figure 4D). Functional measures of contractility, assessed in a $3 \mathrm{D}$ collagen gel system, demonstrated that both baseline and TGF- $\beta 1$-induced contractility were inhibited by fasudil (Figure 4E). Together, these data indicated that myofibroblast differentiation (as measured by biochemical, morphologic, and functional parameters) induced by both soluble (TGF- $\beta 1$ ) and matrix (stiffness) signals are inhibited by fasudil.

Fasudil inbibits myofibroblast differentiation by prevention of MKL1 nuclear translocation. To determine the role of MKL1 in fasudil-modulated myofibroblast differentiation in response to TGF- $\beta 1$ and/or matrix stiffening, we either overexpressed nuclear MKL1 or added 
A Time (h) $\quad 0 \begin{array}{lllllll}0 & 1 & 3 & 5 & 8 & 16 & 24\end{array}$

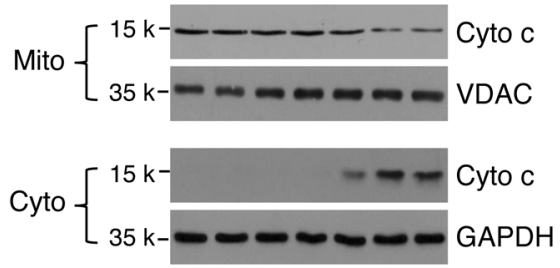

B

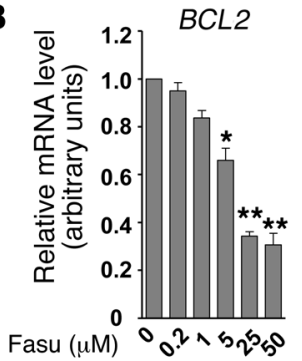

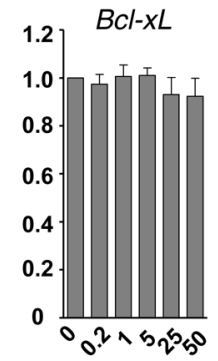

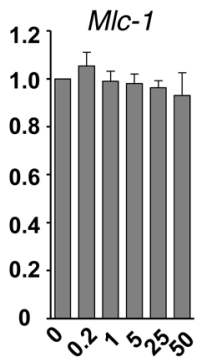

C

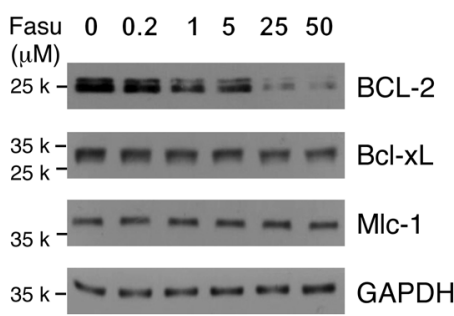

D
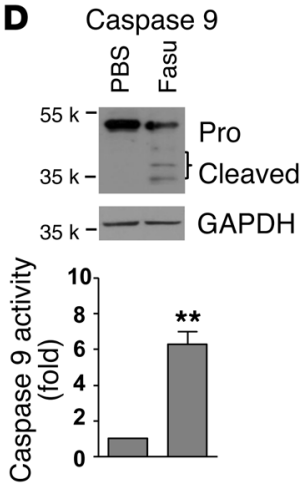

Caspase 3 兽茎

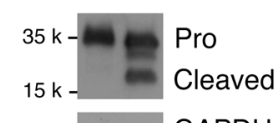

$E$

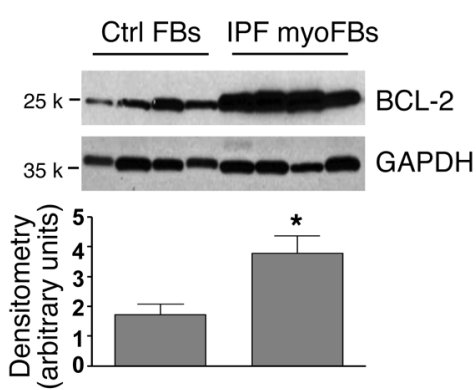

\section{Figure 2}

Fasudil promotes myofibroblast apoptosis by BCL-2 downregulation. (A) IPF lung myofibroblasts were treated with $25 \mu \mathrm{M}$ fasudil for 24 hours. Cell lysates were collected at the indicated time points. Release of cytochrome $c$ (Cyto $\mathrm{c}$ ) from mitochondria was evaluated by a change in the levels of cytochrome $c$ in the mitochondrial (Mito) and cytoplasmic (Cyto) fractions. VDAC and GAPDH were used as loading controls for mitochondrial and cytoplasmic proteins, respectively. (B) IPF lung myofibroblasts were treated with $0-50 \mu \mathrm{M}$ fasudil for 24 hours. Relative levels of $B C L 2$, Bcl$x L$, and MIc-1 mRNA were determined by real-time RT-PCR. 18s rRNA was used as internal reference control. Data are mean \pm SD of 3 separate experiments. (C) Protein levels of BCL-2, Bcl-xL, and Mlc-1 (24 hours after treatment) were determined by immunoblot. GAPDH was used as loading control. (D) IPF lung myofibroblasts were treated with $25 \mu \mathrm{M}$ fasudil or an equal volume of PBS for 24 hours. Cleavage of caspase 9 and caspase 3 was determined by immunoblot analysis. GAPDH was used as loading control. Caspase activities were measured with a colorimetric assay. Data are mean \pm SD of 3 separate experiments. (E) Constitutive expression of BCL-2 protein in IPF myofibroblasts and non-IPF control fibroblasts were determined by immunoblot analyses. GAPDH was used as loading control. Densitometry was performed using ImageJ. Relative density of BCL-2 was normalized to GAPDH. Data are mean \pm SD $\left(n=4\right.$ per group). ${ }^{\star} P<0.05,{ }^{* \star} P<0.01$.

jasplakinolide to force MKL1 in the nucleus (20). Forced nuclear localization of MKL1 abrogates fasudil inhibition of $\alpha$-SMA expression in lung fibroblasts in response to TGF- $\beta 1$ and matrix stiffening (Supplemental Figure 5A). Furthermore, inhibition of actin polymerization by latrunculin $B$ or disruption of MKL1-SRF interaction by CCG- 1423 blocked TGF- $\beta 1$ - and matrix stiffeninginduced $\alpha$-SMA expression in lung fibroblasts (Supplemental Figure 5B), similar to the effects observed with fasudil. Together, these data suggest that fasudil inhibits lung fibroblast-to-myofibroblast differentiation by blocking MKL1 nuclear translocation.

RhoA/ROCK signaling is activated in buman IPF and in bleomycin injury-induced mouse lung fibrosis. $\mathrm{MLC}_{20}$ and myosin phosphatase target subunit 1 (MYPT-1) are ROCK-specific substrates (42-44). Previously, we reported that phosphorylation of $\mathrm{MLC}_{20}$ at serine 19 residue is increased in the fibroblastic foci in human IPF (45). Here, using an antibody that detects $\mathrm{MLC}_{20}$ only when dually phosphorylated at threonine 18 and serine 19, we demonstrated dual phosphorylation of $\mathrm{MLC}_{20}$ in fibroblastic foci of human IPF (Figure 5A). Additionally, we demonstrated increased MYPT-1 phosphorylation in regions of active fibrosis in mice subjected to bleomycin-induced lung injury (Figure 5A). Fasudil $(25 \mathrm{mg} / \mathrm{kg} / \mathrm{d}$ intraperitoneally) blocked MYPT-1 phosphorylation in fibrotic mouse lungs (Figure 5A), validating the inhibitory effect of fasudil on ROCK signaling in bleomycin-induced mouse lung fibrosis. (Myo)fibroblasts isolated from IPF lungs and lungs of mice after bleomycin injury demonstrated high constitutive ROCK activity, as indicated by ELISA-based ROCK activity assay, in comparison to their respective controls (Figure 5B). Immunoblot analysis for phosphorylation of ezrin ${ }^{\text {Thr567 }} /$ radixin $^{\text {Thr564 }} /$ moesin $^{\text {Thr558 }}$ (ERM), a group of ROCK-specific substrates that function as linkers between plasma membrane and actin cytoskeleton $(42,46)$, demonstrated high baseline levels of phosphorylated ERM in fibrotic lung fibroblasts from mice and humans (Figure 5C). Rhotekin pulldown assays, based on affinity precipitation of GTP-bound (i.e., active) RhoA using glutathione S-transferase-rhotekin (GSTRBD) fusion proteins and immunoblot analysis, demonstrated that (myo)fibroblasts isolated from fibrotic lungs expressed higher levels of active RhoA than cells isolated from control uninjured lungs (Figure 5D). These data indicate that RhoA/ROCK signaling is activated in lung fibrosis in vivo as well as in (myo)fibroblasts isolated from fibrotic lungs.

Fasudil treatment or MKL1 deficiency protects from bleomycin injuryinduced lung fibrosis. To determine whether fasudil protects mice from bleomycin injury-induced lung fibrosis and whether the protective effect is associated with the blockade of MKL1-mediated intrinsic mechanotransduction, C57BL/6 WT, $\mathrm{Mkl1}^{-/-}$, and $\mathrm{Mkl1}^{+/+}$ 
A
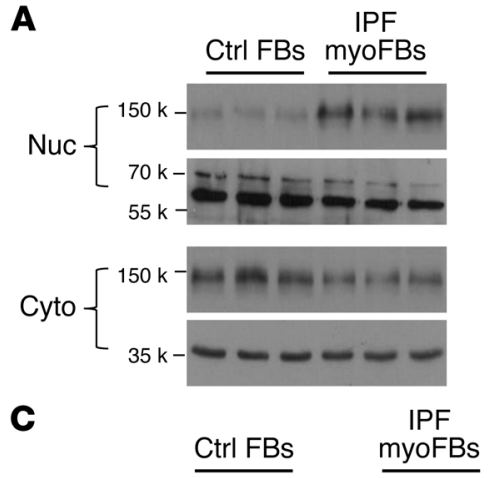

B

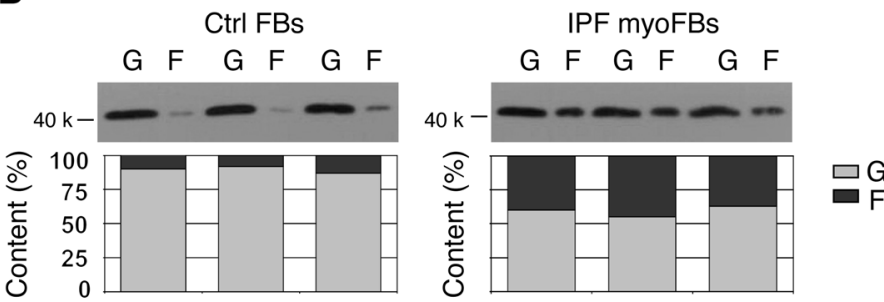

D
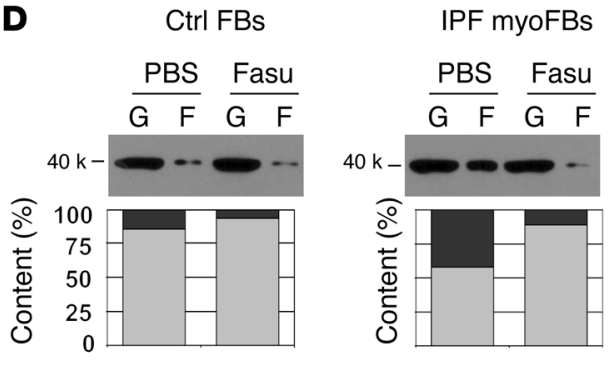

$\mathbf{F}$

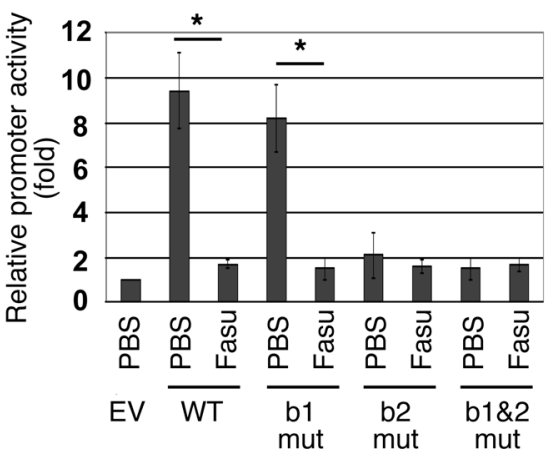

E

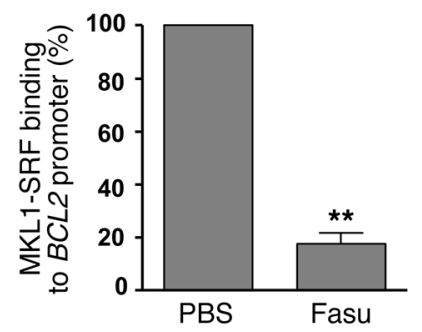

G

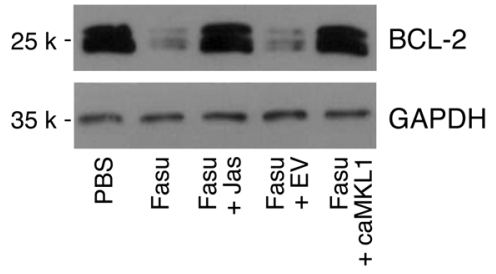

$\square \mathrm{G}$

H

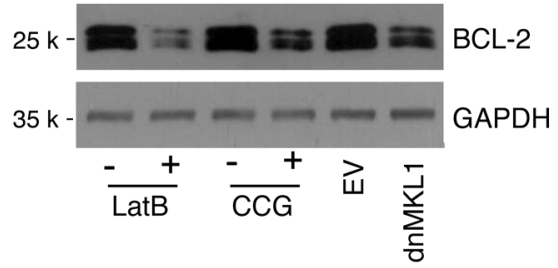

Figure 3

Fasudil downregulation of BCL-2 expression occurs by deactivation of MKL1-mediated intrinsic mechanotransduction. (A) Subcellular distribution of MKL1 in IPF lung myofibroblasts and control fibroblasts, determined by subcellular fractionation followed by immunoblot. Lamin A/C and GAPDH were used as loading controls for nucleic and cytoplasmic proteins, respectively. (B) F-actin (F) and G-actin (G) content in IPF lung fibroblasts and control fibroblasts, determined by immunoblot and densitometric analysis. (C and D) IPF myofibroblasts and control fibroblasts were treated with PBS or $25 \mu \mathrm{M}$ fasudil for 24 hours. (C) MKL1 subcellular localization, determined as in A. (D) F-actin and G-actin content, determined as in B. (E) Binding of MKL1-SRF complex to BCL2 promoter was quantified by quantitative ChIP. Data are mean \pm SD of 3 separate experiments. (F) WT and 3 mutated human BCL2 promoters: CArG box1 (b1 mut), CArG box2 (b2 mut), or both box1 and box2 (b1\&2 mut). Promoter reporters were transfected into IPF myofibroblasts and treated with PBS or $25 \mu \mathrm{M}$ fasudil for 24 hours. Promoter activity was determined by luciferase assay. Data are mean \pm SD of 3 separate experiments, each performed in triplicate. EV, empty vector. (G) IPF myofibroblasts were treated with PBS or $25 \mu \mathrm{M}$ fasudil in the presence or absence of $200 \mathrm{nM}$ jasplakinolide (Jas) for 24 hours. A subset of cells was transfected with constitutively active MKL1 (caMKL1) or empty vector before fasudil treatment. Protein levels of BCL-2 and GAPDH were determined by immunoblot. (H) IPF myofibroblasts were cultured in the presence or absence of $1 \mu \mathrm{M}$ latrunculin B (LatB) or $1 \mu \mathrm{M} \mathrm{CCG-1423}$ (CCG) for 24 hours. A subset of cells was transfected with dnMKL1-expressing plasmid or empty vector. Protein levels of BCL-2 and GAPDH were determined by immunoblot. ${ }^{\star} P<0.05,{ }^{* \star} P<0.01$.

mice were subjected to lung injury by intratracheal instillation of bleomycin. In parallel, a subgroup of C57BL/6 mice received fasudil or PBS control daily via intraperitoneal injections; to more specifically target the fibrotic versus the early inflammatory response, fasudil was administered beginning 14 days after bleomycin injury for a period of 2 weeks. Our data showed that treatment with fasudil during the postinflammatory fibrotic phase of lung repair abrogated fibrotic responses, as assessed by trichrome staining of the lung for collagen, whole-lung hydroxyproline content, and induction of $\alpha$-SMA protein in lung homogenates (Figure 6, A-C). In contrast to $\mathrm{Mkl1^{+/+ }}$ mice, $\mathrm{Mkl1^{-/ }}$ mice demonstrated significantly reduced fibrotic responses, as measured by the same endpoints (Figure 6, D-F). Collectively, these data suggest that fasudil mediates antifibrotic effects even when administered to mice with established fibrosis, likely by disruption of myofibroblast contractility and MKL1-mediated intrinsic mechanotransduction. 


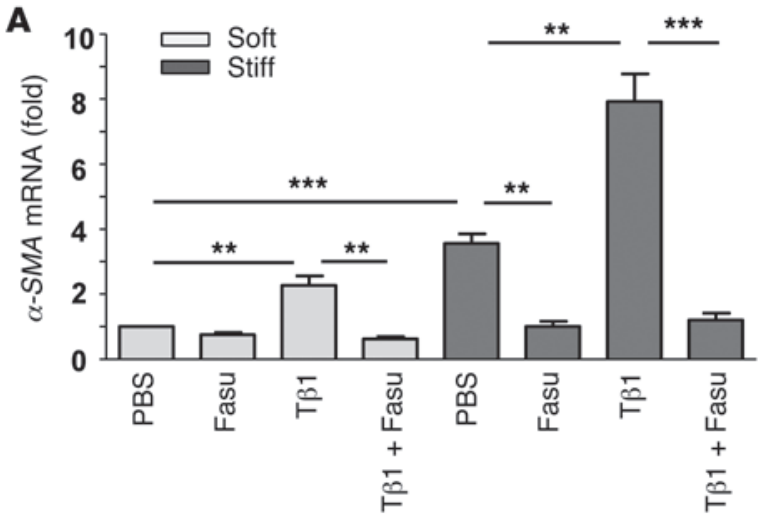

B

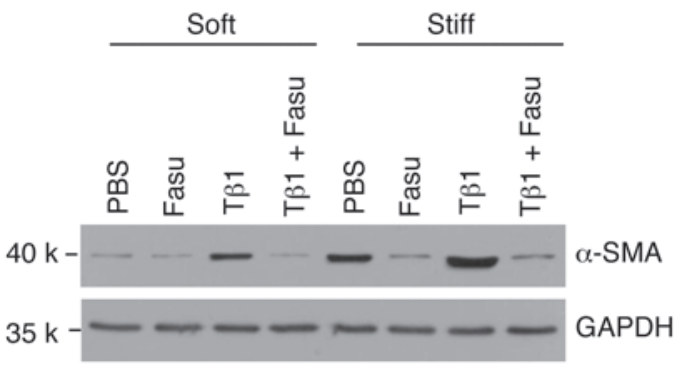

C
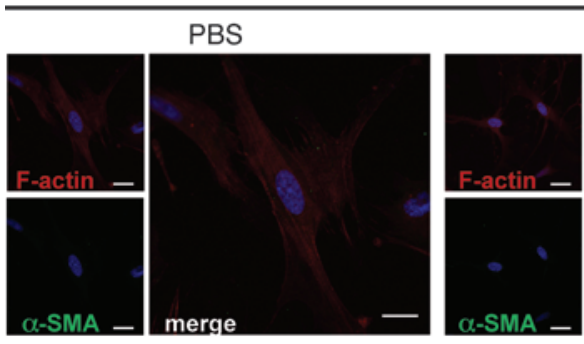

Fasu

Soft
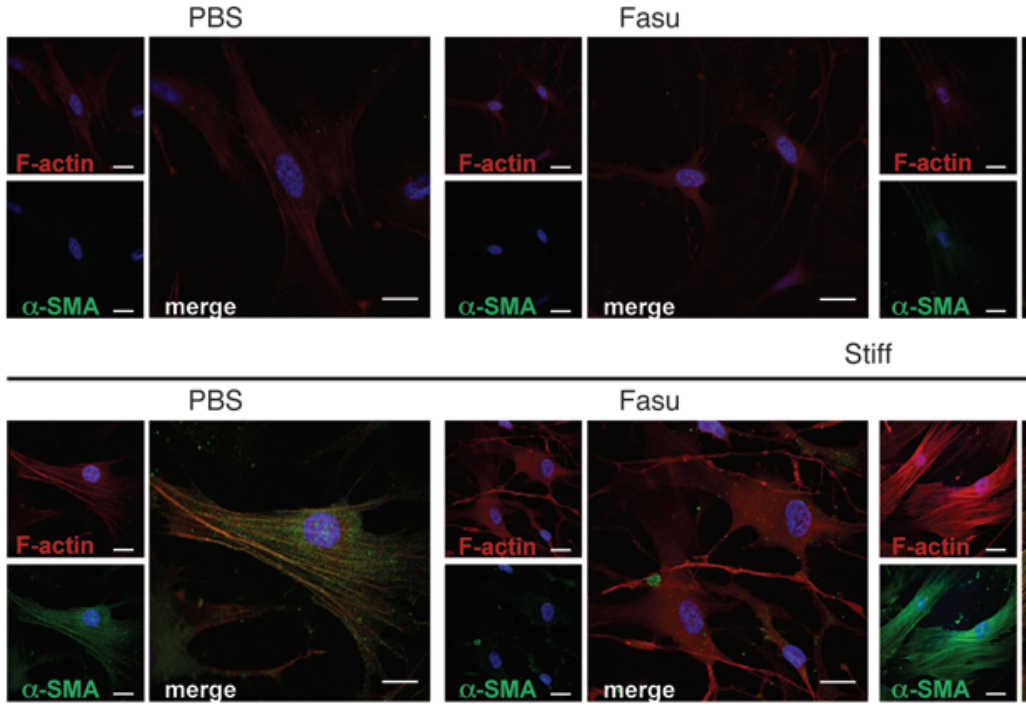

$\mathrm{T} \beta 1$

$\mathrm{T} \beta 1+\mathrm{Fasu}$
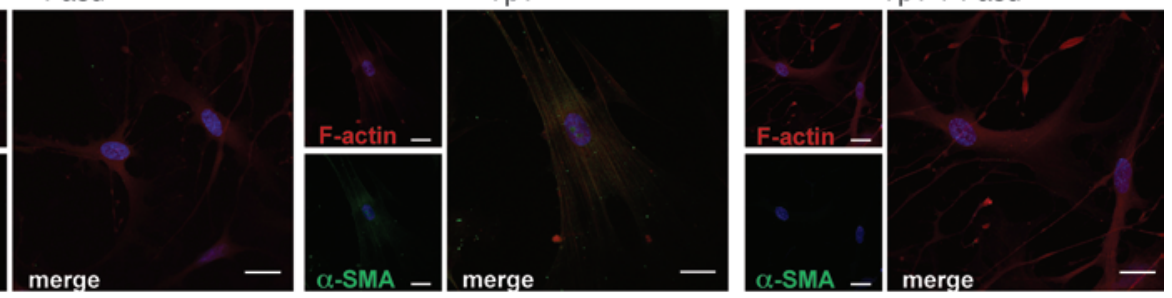

Stiff

D
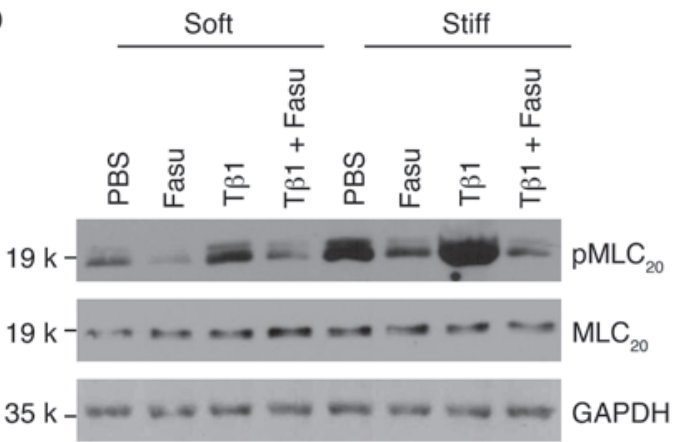

Fasu
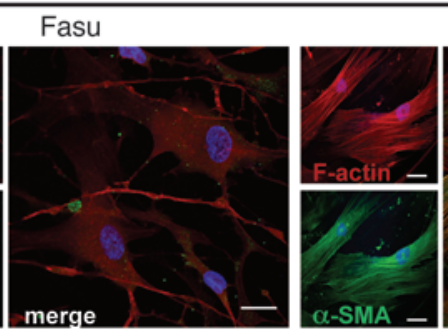

E
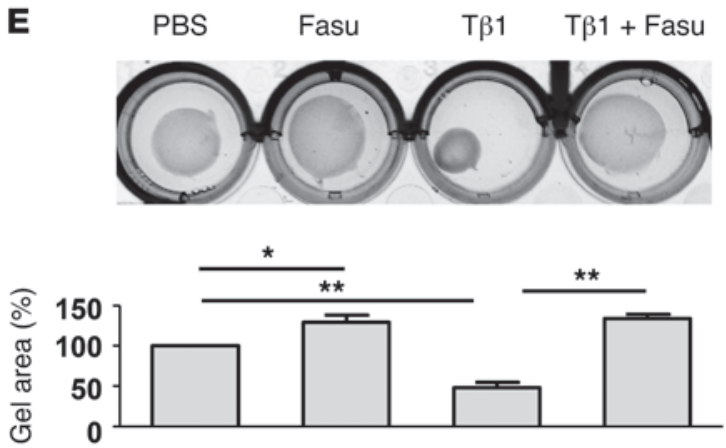

Figure 4

Fasudil inhibits lung fibroblast-to-myofibroblast differentiation in response to TGF- $\beta 1$ and matrix stiffening. (A) Normal human lung fibroblasts were cultured on soft $(0.5 \mathrm{kPa})$ and stiff $(20 \mathrm{kPa})$ PA gels in the presence of PBS, $25 \mu \mathrm{M}$ fasudil, $4 \mathrm{ng} / \mathrm{ml}$ TGF- $\beta 1$, or fasudil and TGF- $\beta 1$ in combination for 24 hours. Relative levels of $\alpha$-SMA mRNA were determined by real-time RT-PCR. 18s rRNA was used as internal reference control. Bar graphs are the means \pm SD of 3 separate experiments. (B) Protein levels of $\alpha$-SMA and GAPDH were determined by immunoblot. (C) Incorporation of $\alpha$-SMA into stress fibers was determined by confocal immunofluorescent double staining for $\alpha$-SMA (green) and F-actin (red). Nuclei were stained by DAPI (blue). Scale bars: $50 \mu \mathrm{m}$. (D) Expression of phosphorylated (p-) and total $\mathrm{MLC}_{20}$ and GAPDH was determined by immunoblot. (E) Fibroblast contractility was assessed by a 3D collagen gel-based assay. Bar graphs are the means \pm SD of 3 separate experiments, each performed in triplicate. ${ }^{*} P<0.05,{ }^{* *} P<0.01,{ }^{* \star *} P<0.001$. 


\section{Discussion}

Myofibroblasts are key effector cells in fibrogenic processes and have been proposed to derive from multiple origins $(47,48)$. Regardless of origin, a therapeutic strategy that exploits the unique biomechanical contractile signaling of myofibroblasts and their prolonged survival in injured tissues may be most effective in advanced fibrotic diseases. In this study, we demonstrated for the first time that therapeutic targeting of the mechanosensitive Rho/ ROCK pathway concordantly altered actin cytoskeletal dynamics, MKL1 translocation/activation, and regulation of profibrotic and prosurvival genes (Figure 7).

Specifically, we showed that the ROCK pathway mediated myofibroblast contractility, differentiation, and survival. Blockade of this pathway by fasudil prevented lung fibroblast differentiation into myofibroblasts. Importantly, we demonstrated both in vitro and in vivo that disruption of myofibroblast contractility induced preexisting lung myofibroblasts to undergo apoptosis. Fasudil's ability to induce apoptosis was selective to myofibroblasts (compared with nonmyofibroblasts), supporting an "intrinsic dependence" on the actomyosin system for maintaining survival of these differentiated cells. Our studies suggest that this survival signaling pathway is controlled by actin dynamics-dependent MKL1 nuclear translocation in response to biomechanical and biochemical fibrogenic stimuli involving matrix stiffening and TGF- $\beta 1$, respectively. ROCK inhibition depolymerizes actin cytoskeleton, decreases myofibroblast contractility, and deactivates MKL1 nuclear signaling, leading to downregulation of BCL-2 expression and subsequent activation of the mitochondria-dependent intrinsic apoptosis pathway (Figure 7). Our findings suggest that targeting myofibroblast contractility by use of a pharmacologic ROCK inhibitor, such as fasudil, may offer a novel approach for effectively treating persistent/progressive fibrosis by both preventing fibroblast-to-myofibroblast differentiation and inducing preexisting myofibroblasts to undergo apoptosis.

An intriguing finding in our studies was the marked susceptibility of differentiated myofibroblasts to ROCK inhibition-induced apoptosis. Fasudil (at relatively low concentrations, 5-25 $\mu \mathrm{M}$ ) induced myofibroblasts to undergo apoptosis, whereas control nonmyofibroblasts were resistant. This differential susceptibility to apoptosis appears to be linked with more mature, polymerized actin cytoskeleton, as evidenced by higher F-actin/G-actin ratios, in myofibroblasts. IPF lung myofibroblasts were found to have higher F-actin content at the basal level, with $37 \%-45 \%$ of total actin in the filamentous form, whereas control lung fibroblasts had a baseline F-actin content of $8 \%-17 \%$. ROCK inhibition markedly decreased F-actin content in myofibroblasts, from an average of $42 \%$ to an average of $7 \%$. In contrast, fasudil only slightly decreased F-actin content in normal fibroblasts, from $14 \%$ to $6 \%$. Cellular apoptosis related to changes in actin dynamics has been previously reported (49-51), although the mechanisms have not been clearly elucidated. Our present findings suggest that changes in the F-actin/G-actin ratio provide a sensory mechanism for apoptotic signaling in lung myofibroblasts. Importantly, we demonstrated a key role for MKL1 in transducing actin cytoskeletal signals to the mitochondria-mediated apoptosis pathway. ROCK inhibition deactivated MKL1 nuclear signaling in lung myofibroblasts, resulting in downregulation of BCL-2 expression and initiation of the intrinsic apoptotic cascade.

MKL1 has been reported to regulate apoptosis of myoepithelial cells, a type of specialized epithelial cells with contractile characteristics similar to those of smooth muscle cells (52). Genetic deletion of Mkl1 causes myoepithelial cell apoptosis and failure to form normal lobular-alveolar structures of mammary glands during lactation in female mice (52). Conversely, overexpression of MKL1 inhibits apoptosis, with attendant inhibition of caspase activation (53). These prior reports and the findings of the current study strongly implicate MKL1 as a determinant of the fate of differentiated cells that contain mature actomyosin contractile elements. It has been reported that MKL1 mediates TGF- $\beta 1$-induced epithelial-mesenchymal transition (EMT) by dissociation of cellcell contacts and induction of actin cytoskeletal reorganization (33). MKL1 is thought to be essential for completing the myogenic phase in an EMT process (36). These findings suggest that blocking MKL1-mediated signaling may have additional antifibrotic benefits by inhibiting EMT.

Cofilin, a member of the cofilin/ADF family, promotes the depolymerization of F-actin and is involved in the recycling of monomeric G-actin $(54,55)$. Dephosphorylated (active) cofilin targets mitochondria to induce loss of mitochondrial membrane potential, cytochrome $c$ release, and cellular apoptosis (49, $56,57)$. We examined whether a cofilin-dependent apoptosis mechanism is involved in ROCK inhibition-induced myofibroblast programmed death. We found that fasudil changed neither phosphorylation of cofilin nor its subcellular localization in lung myofibroblasts (Supplemental Figure 6). Furthermore, our data showed that fasudil-induced cytochrome $c$ release occurred 8-24 hours after treatment, in contrast to a previous report that cofilin-mediated cytochrome $c$ release occurred within 2-4 hours (49). Together, these data suggest that the fasudil-induced myofibroblast apoptosis reported herein is independent of a cofilinmediated mechanism. Additionally, the relatively longer period of time required for cytochrome $c$ release from mitochondria after fasudil treatment ( $\geq 8$ hours) is consistent with our data supporting fasudil regulation of myofibroblast apoptosis by downregulation of BCL2 gene expression.

Resolution of tissue repair after tissue injury involves myofibroblast clearance (10). Elimination and/or deactivation of myofibroblasts can occur through apoptosis, senescence, and/or regression to a more quiescent phenotype $(7-9,58)$. Although regression to an inactive precursor cell may limit the activity of myofibroblasts, they do not completely eliminate the potential for reactivation. For example, in a carbon tetrachloride-induced mouse model of liver fibrosis, HSC-derived myofibroblasts that regressed to a quiescent state were more rapidly reactivated (compared with native HSCs) by subsequent insults, resulting in more robust liver fibrosis (7). It is possible that myofibroblast regression to a quiescent phenotype may serve as an intermediate step toward resolution of fibrosis. In mature myofibroblasts, the release of mechanical tension triggers apoptosis in vitro and in vivo (58-60). In a normal woundhealing process, the release of mechanical tension may occur by restoration of normal ECM composition and mechanics. This would essentially eliminate the sustained biomechanical signals, both intrinsic and extrinsic, that would otherwise maintain the myofibroblastic phenotype. Thus, a cellular phenotype (contractility) and a matrix property (stiffness) reciprocally regulate each other and may serve as feed-forward mechanisms to sustain fibrotic responses. In persistent/progressive fibrosis, such as IPF, mechanisms involved in matrix turnover have been dampened (61); this may impair the restoration of normal ECM composition and biomechanical tension. The findings from 

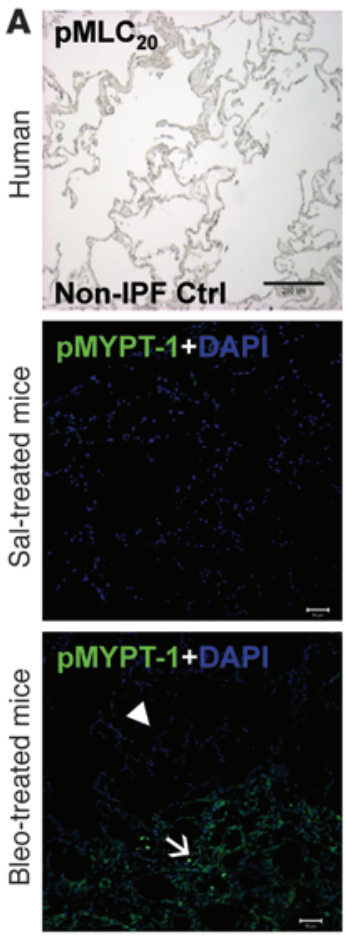

C
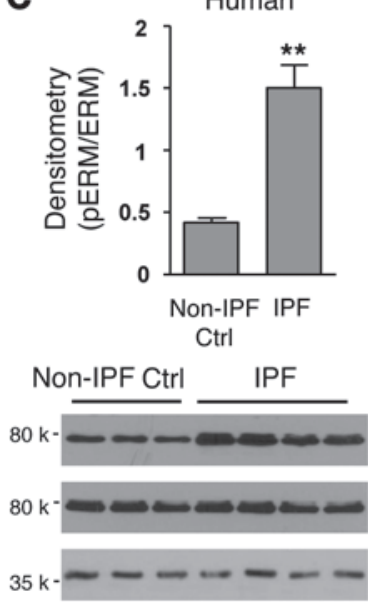
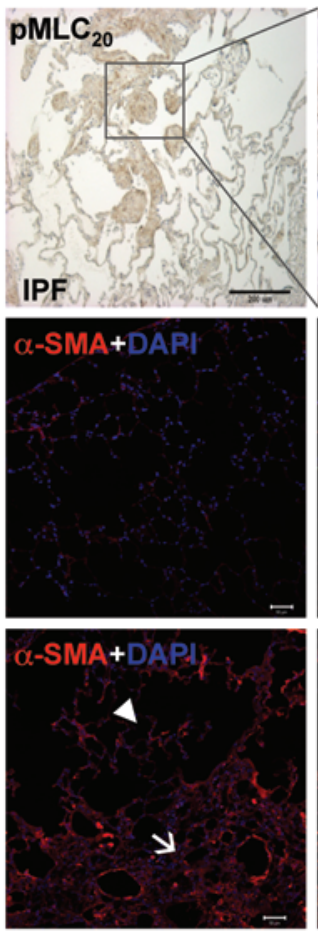

Mouse
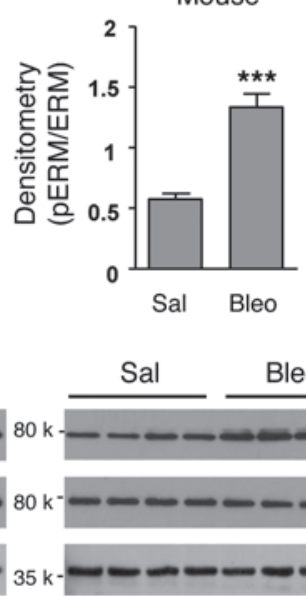
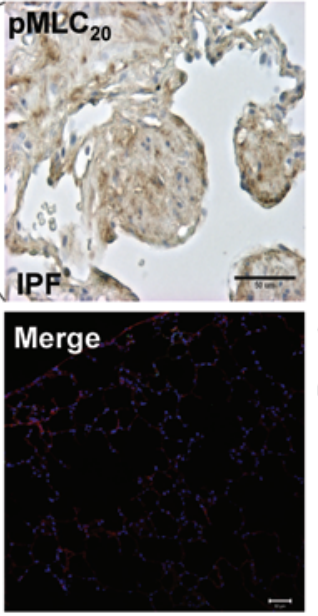

Merge

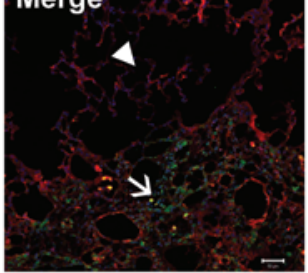

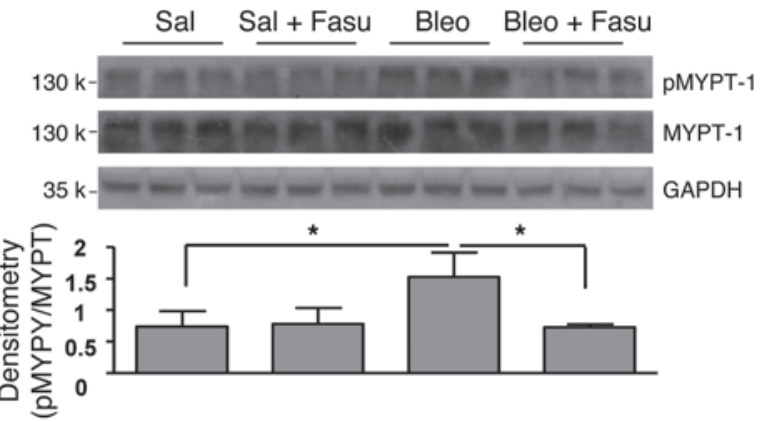

B

D
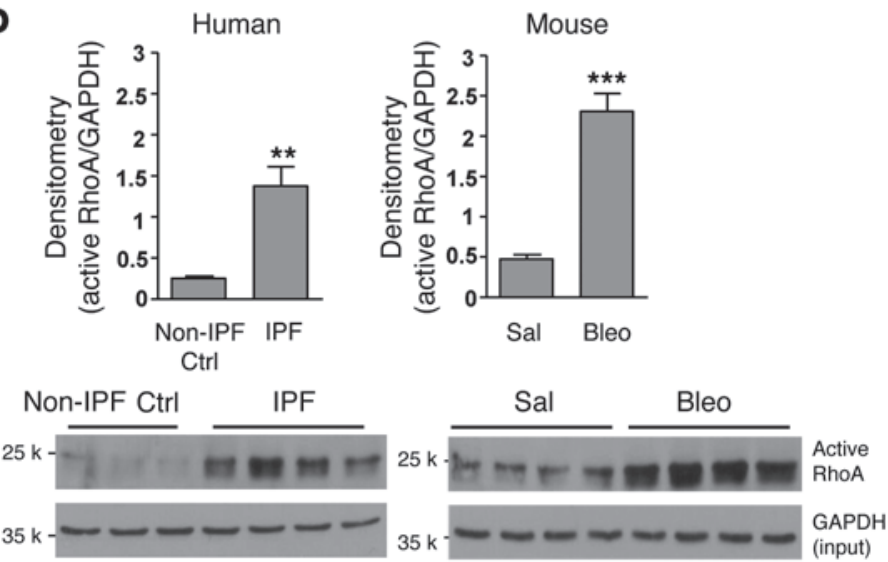

GAPDH
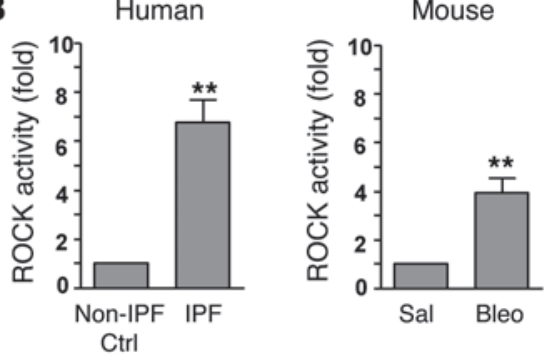

\section{Figure 5}

RhoA/ROCK signaling is enhanced in regions of active fibrosis in human IPF and in mice subjected to bleomycin-induced lung injury, as well as in (myo)fibroblasts isolated from fibrotic lungs. (A) Paraffin-embedded lung tissue sections from non-IPF control subjects $(n=3)$ and patients with IPF $(n=5)$ were subjected to immunohistochemical staining for phosphorylated $\mathrm{MLC}_{20}$. Frozen mouse lung tissue sections from saline- or bleomycin-treated mice ( $n=5$ per group) were subjected to double staining for $\alpha$-SMA (red) and phosphorylated MYPT-1 (green). Nuclei were stained by DAPI. Confocal microscopic images are overlaid to show the correlation of MYPT-1 phosphorylation with fibrosis. Arrowheads denote relatively normal lung area; arrows denote fibrotic lung area. Scale bars: $50 \mu \mathrm{m} ; 200 \mu \mathrm{m}$ (higher-magnification view of boxed region in IPF panel). Levels of phosphorylated and total MYPT-1 in mouse lung homogenates were also determined by immunoblot. GAPDH was used for loading control. Data are mean \pm SD ( $n=5$ per group). (B) (Myo)fibroblasts isolated from lungs of patients with IPF $(n=8)$, non-IPF control subjects $(n=6)$, bleomycin-treated mice $(n=8)$, and saline-treated mice $(n=8)$ were subjected to ROCK activity assays using a colorimetric approach. Data are mean \pm SD. (C) Relative protein levels of phosphorylated versus total ERM in isolated (myo) fibroblasts by densitometric analysis. Data are mean $\pm \operatorname{SD}(n=6$ or 8$)$. Representative immunoblots are shown. (D) Level of active RhoA (normalized by GAPDH) in isolated (myo)fibroblasts by densitometric analysis. Data are mean \pm SD ( $n=8$ per group). Representative immunoblots are shown. ${ }^{*} P<0.05,{ }^{* *} P<0.01,{ }^{* * *} P<0.001$. 
A

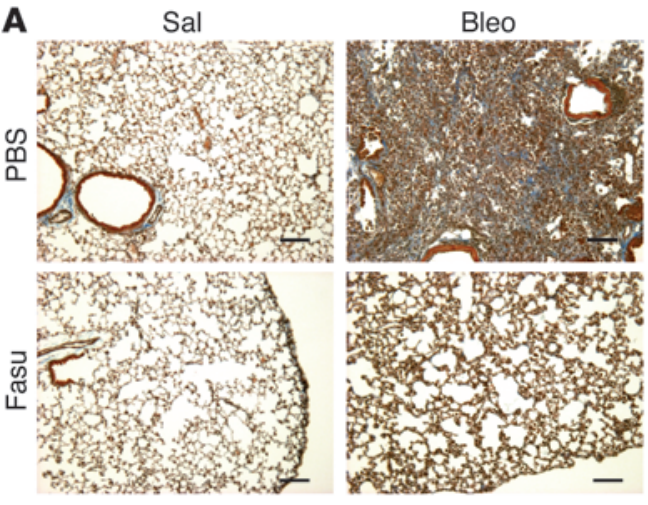

B

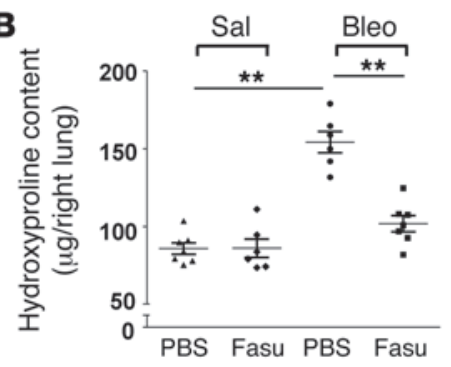

C

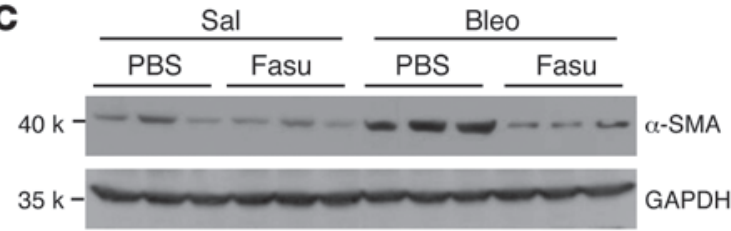

D

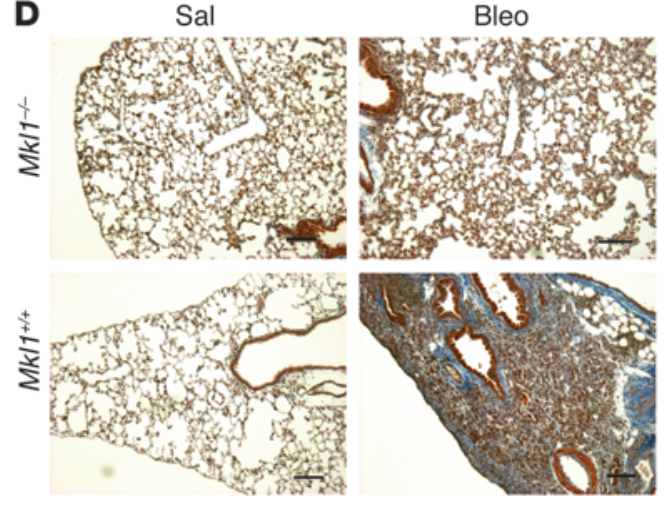

$\mathbf{E}$
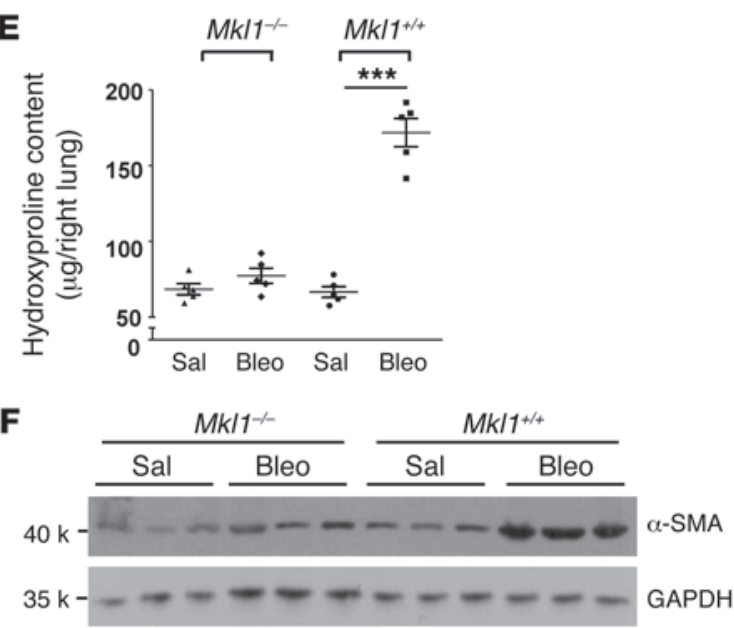

Figure 6

Postinflammatory treatment with fasudil or genetic deletion of $M k / 1$ protects mice from bleomycin injury-induced lung fibrosis. (A-C) C57BL/6 mice were administered bleomycin intratracheally at a dose of $5 \mathrm{U} / \mathrm{kg}$. Control mice were given an equal volume of saline. At 14 days, mice were administered fasudil intraperitoneally at a dose of $25 \mathrm{mg} / \mathrm{kg} / \mathrm{d}$ over 14 days. Control mice were injected with an equal volume of PBS. Lung tissues were harvested at 28 days and subjected to Masson trichrome staining for collagen (A), hydroxyproline content assay (B), and immunoblot analysis for $\alpha$-SMA expression (C). (D-F) $M k / 1^{-/-}$and $M k / 1^{+/+}$mice were administered bleomycin or saline as above. Mouse lung tissues were harvested at 28 days and subjected to Masson trichrome staining (D), hydroxyproline content assay (E), and $\alpha$-SMA expression analysis (F). Graphs are mean \pm SD $(n=5-7)$. Representative immunoblots are shown. Scale bars: $100 \mu \mathrm{m} .{ }^{* \star} P<0.01 ;{ }^{* \star *} P<0.001$.

the present study suggest that a cell-based approach targeting myofibroblast contractility and mechanotransduction offer an alternative to matrix targeting for tension release-induced myofibroblast apoptosis.

There is accumulating evidence in support of a role for both TGF- $\beta 1$ (62) and biophysical properties of the ECM (63) in regulating the myofibroblastic phenotype. Our present data support the concept that MKL1 serves as a master control switch that regulates both TGF- $\beta 1$ - and matrix stiffening-induced fibroblast-to-myofibroblast differentiation. This is consistent with studies demonstrating that MKL1 transduces TGF- $\beta$ signals to the nucleus via its capacity to physically associate with receptor-activated Smads $(33,36,64)$. In the nucleus, Smads associate with MKL1, facilitating the binding of a MKL1/Smad complex to transcriptional regulatory elements that control transcription of a subset of genes encoding contractile SMC proteins and fibrosis-associated proteins. Our data indicated that disruption of fibroblast contraction or blocking MKL1mediated intrinsic mechanotransduction is sufficient to inhibit TGF- $\beta 1$ - and/or matrix stiffness-induced fibroblast-to-myofibroblast differentiation. Additionally, mice deficient in Mkl1 were protected from bleomycin-induced lung fibrosis. Together, our findings reveal an indispensable role of MKL1-mediated biomechanical signaling in the regulation of TGF- $\beta 1$-induced myofibroblast differentiation and survival as well as in injuryinduced lung fibrosis.

Interestingly, our data also suggest that fasudil may mediate protective effects on alveolar epithelial cell apoptosis during the late reparative phase of injury in the murine model of bleomycin-induced lung fibrosis. Although this observation may be related to direct effects of fasudil on the epithelium (such as EMT, as discussed above), other potential "indirect" mecha- 


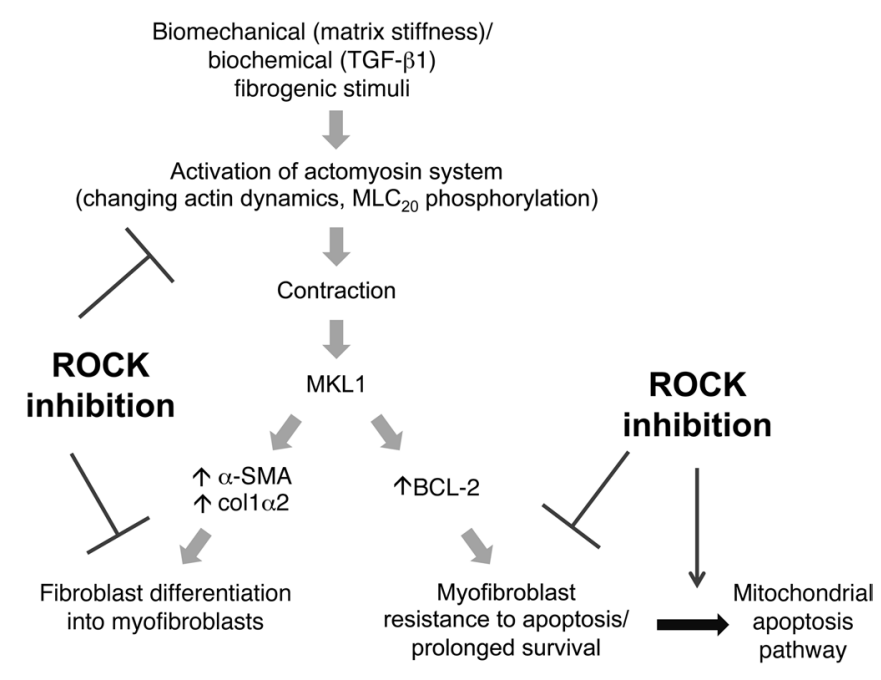

nisms may involve the predicted downregulation of myofibroblast-generated paracrine mediators known to induce alveolar epithelial cell apoptosis. Such myofibroblast-derived mediators include active TGF- $\beta 1$ derived from myofibroblast contractile force-induced latent TGF- $\beta 1$ activation $(16,17)$, angiotensinII (65), Fas ligand (66), and reactive oxygen species (67). These direct or indirect protective effects on alveolar epithelial cells add further support for targeting the ROCK pathway in established lung fibrosis.

Antifibrotic therapies targeting the recalcitrant myofibroblast are limited. Acquisition of contractility is crucial not only for the emergence of the myofibroblastic phenotype, but also for its maintenance. Our present data provide strong proof-of-concept that targeting myofibroblast contractility (or the consequent mechanotransduction pathway) offers a promising and novel approach to hinder, or possibly reverse, persistent/progressive fibrosis by both prevention of myofibroblast differentiation from its precursors and induction of preexisting myofibroblasts to undergo apoptosis.

\section{Methods}

Lung fibroblast isolation, culture, transfection, and treatment. Human lung (myo)fibroblasts were established from tissue samples from patients undergoing lung transplantation for IPF or failed donors as approved by Human Studies Committee of the University of Alabama at Birmingham. For lung fibroblast isolation, culture, transfection, sorting, and treatment, see Supplemental Methods.

TUNEL and immunofluorescence double-labeling assay. To combine TUNEL staining with immunofluorescence staining for specific antigens, TUNEL staining was carried out first using a TdT-fluor in situ apoptosis detection kit (Trevigen), followed by exposure with one of the primary antibodies for 24 hours. Fluorochrome-conjugated secondary antibodies were used according to the manufacturer's recommendation. Nuclei were stained with DAPI.

Confocal laser scanning microscopy. Fluorescent signals were detected using a confocal laser-scanning microscope Zeiss LSM510 confocal microscope equipped with a digital color camera (AxioCam). All fluorescent images were generated using sequential laser scanning with only the corresponding single-wavelength laser line, activated using acoustooptical tunable filters to avoid cross-detection of either one of the fluorescence channels.

\section{Figure 7}

Inhibition of myofibroblastic phenotype and lung fibrosis by targeting (myo)fibroblast contractility and MKL1-mediated intrinsic mechanotransduction with ROCK inhibitors. In response to extracellular biomechanical (e.g., matrix stiffness) and biochemical (e.g., active TGF- $\beta 1$ ) stimuli, lung fibroblasts undergo actin cytoskeleton remodeling and activation of the actomyosin contractile system, resulting in MKL1 translocation from cytoplasm to nucleus, where it activates fibrotic genes that specify myofibroblast differentiation. Inhibition of ROCK blocks actin cytoskeletal reorganization, fibroblast acquisition of contractile activity, and MKL1 nuclear translocation, preventing fibroblast-to-myofibroblast differentiation. On the other hand, ROCK inhibition disrupts actin cytoskeleton required for myofibroblast contractility in preexisting myofibroblasts. This deactivates constitutively activated MKL1 nuclear signal in myofibroblasts, resulting in downregulation of the antiapoptotic protein BCL-2 and activation of the intrinsic apoptotic pathway.

Quantitative ChIP assay. Fibroblasts $\left(1 \times 10^{6}\right.$ cells) were treated with $1 \%$ formaldehyde for 10 minutes at $37^{\circ} \mathrm{C}$ to cross-link histones to DNA. The cross-linked chromatin was sonicated to shear chromatin fragments of 200-1,000 bp. A portion of sheared chromatin was reversed at $65^{\circ} \mathrm{C}$ for 4 hours, and cross-linked DNA was purified by phenol/chloroform extraction. The DNA was saved and used as an internal reference control in the subsequent real-time PCR reactions. The rest of the sonicated chromatin was immunoprecipitated with anti-SRF antibody at a concentration recommended by the manufacturer, while the negative control was immunoprecipitated with no antibody. Immunocomplexes were recovered with Protein A agarose beads. Cross-links were reversed and treated by proteinase $\mathrm{K}$ to remove protein from the DNA. DNA was purified by phenol/ chloroform extraction. Real-time PCR was performed to quantify SRFbinding $B C L 2$ promoter fragments using the following primers: forward, 5'-CGCCGCGCAGGACCAGGAGGAGGAGAAA-3'; reverse, 5'-CGGCGAGGGGTGGGGAGAAGGAGGTGGT-3'.

Animals and experimental protocol. Animal use and bleomycin protocols were approved by the Institutional Animal Care and Use Committee of the University of Alabama at Birmingham. 6- to 8-week-old pathogen-free female mice were used in this study. Bleomycin sulphate (Almirall) was dissolved in sterile saline solution and intratracheally instilled into mice by a Stepper Repetitive Pipette (Tridak) as a single dose of $0.08 \mathrm{mg}$ in $50 \mu \mathrm{l}$ saline solution per animal $(5 \mathrm{U} / \mathrm{kg} \mathrm{BW})$. Control mice received $50 \mu \mathrm{l} \mathrm{saline.}$ For fasudil treatment, a dosage of $25 \mathrm{mg} / \mathrm{kg} \mathrm{BW} / \mathrm{d}$ over 14 days or an equal volume of PBS was injected intraperitoneally into mice beginning 14 days after bleomycin administration. Mice were sacrificed at 28 days. Lung tissues were excised and inflated with OCT or $4 \%(\mathrm{~m} / \mathrm{v})$ paraformaldehyde in PBS for immunofluorescent or immunohistochemical analyses.

To prepare for lung homogenates, fresh lung tissues were transferred to prechilled tubes containing T-PER (Thermo Scientific) and Complete Mini Protease Inhibitor Cocktail (Roche) at a proportion of 1 tablet/10 ml T-PER and were homogenized at $4^{\circ} \mathrm{C}$. Homogenates were centrifuged at $9,000 \mathrm{~g}$ for 10 minutes at $4^{\circ} \mathrm{C}$. Supernatants were transferred to clean microcentrifuge tubes. Total protein concentrations in the lung tissue homogenates were determined using a BCA Protein Assay kit (Thermo Scientific).

Statistics. Statistical differences among treatment conditions were determined using 1-way ANOVA and the Newman-Keuls method for multiple comparisons. A $P$ value less than 0.05 was considered significant.

Study approval. Animal studies were conducted in accordance with NIH guidelines for the care and use of laboratory animals and approved by the IACUC of the University of Alabama at Birmingham. Studies involving 
human subjects were approved by the IRB of the University of Alabama at Birmingham, and participants provided written informed consent.

\section{Acknowledgments}

This work was supported in part by NIH grant HL097215, American Heart Association Scientist Development Grant 0835432N, and start-up funds from Department of Medicine, University of Alabama at Birmingham, to Y. Zhou and by NIH grants HL067967 and HL107181 to V.J. Thannickal.
Received for publication September 5, 2012, and accepted in revised form December 13, 2012.

Address correspondence to: Yong Zhou or Victor J. Thannickal, Division of Pulmonary, Allergy, and Critical Care, Department of Medicine, University of Alabama at Birmingham, 1530 3rd Avenue South, THT 422, Birmingham, Alabama 35294-0006, USA. Phone: 205.975.6376; Fax: 205.934.1721; E-mail: yzhou@uab.edu (Y.Zhou), vjthan@uab.edu (V.J. Thannickal).
1. Hinz B, et al. Recent developments in myofibroblast biology: paradigms for connective tissue remodeling. Am J Pathol. 2012;180(4):1340-1355.

2. Noble PW, Barkauskas CE, Jiang D. Pulmonary fibrosis: patterns and perpetrators. J Clin Invest. 2012;122(8):2756-2762.

3. Wynn TA, Ramalingam TR. Mechanisms of fibrosis: therapeutic translation for fibrotic disease. Nat Med. 2012;18(7):1028-1040.

4. Tomasek JJ, Gabbiani G, Hinz B, Chaponnier C, Brown RA. Myofibroblasts and mechano-regulation of connective tissue remodelling. Nat Rev Mol Cell Biol. 2002;3(5):349-363.

5. Gabbiani G. The myofibroblast in wound healing and fibrocontractive diseases. $J$ Pathol. 2003;200(4):500-503

6. Iredale JP, et al. Mechanisms of spontaneous resolution of rat liver fibrosis. Hepatic stellate cell apoptosis and reduced hepatic expression of metalloproteinase inhibitors. J Clin Invest. 1998;102(3):538-549.

7. Kisseleva T, et al. Myofibroblasts revert to an inactive phenotype during regression of liver fibrosis. Proc Natl Acad Sci U S A. 2012;109(24):9448-9453.

8. Desmoulière A, Redard M, Darby I, Gabbiani G. Apoptosis mediates the decrease in cellularity during the transition between granulation tissue and scar. Am J Pathol. 1995;146(1):56-66.

9. Krizhanovsky V, et al. Senescence of activated stellate cells limits liver fibrosis. Cell. 2008;134(4):657-667.

10. Friedman SL. Fibrogenic cell reversion underlies fibrosis regression in liver. Proc Natl Acad Sci U S A. 2012;109(24):9230-9231.

11. Ding Q, et al. New insights into the pathogenesis and treatment of idiopathic pulmonary fibrosis. Drugs. 2011;71(8):981-1001.

12. Hinz B, Gabbiani G. Mechanisms of force generation and transmission by myofibroblasts. Curr Opin Biotechnol. 2003;14(5):538-546.

13. Follonier Castella L, Gabbiani G, McCulloch CA, Hinz B. Regulation of myofibroblast activities: calcium pulls some strings behind the scene. Exp Cell Res. 2010;316(15):2390-2401.

14. Gabbiani G, Majno G. Dupuytren's contracture: fibroblast contraction? An ultrastructural study. Am J Pathol. 1972;66(1):131-146.

15. Desmouliere A, Chaponnier C, Gabbiani G. Tissue repair, contraction, and the myofibroblast. Wound Repair Regen. 2005;13(1):7-12.

16. Wipff PJ, Rifkin DB, Meister JJ, Hinz B. Myofibroblast contraction activates latent TGFbeta1 from the extracellular matrix. J Cell Biol. 2007;179(6):1311-1323.

17. Zhou Y, Hagood JS, Lu B, Merryman WD, MurphyUllrich JE. Thy-1-integrin alphav beta5 interactions inhibit lung fibroblast contraction-induced latent transforming growth factor-beta 1 activation and myofibroblast differentiation.J Biol Chem. 2010;285(29):22382-22393

18. Buscemi L, et al. The single-molecule mechanics of the latent TGF- $\beta 1$ complex. Curr Biol. 2011;21(24):2046-2054.

19 . Shi M, et al. Latent TGF- $\beta$ structure and activation. Nature. 2011;474(7351):343-349.

20. Huang X, et al. Matrix stiffness-induced myofibroblast differentiation is mediated by intrinsic mechanotransduction. Am J Respir Cell Mol Biol. 2012;47(3):340-348.

21. Street CA, Bryan BA. Rho kinase proteins--pleiotropic modulators of cell survival and apoptosis. Anticancer Res. 2011;31(11):3645-3657.

22. Riento K, Ridley AJ. Rocks: multifunctional kinases in cell behaviour. Nat Rev Mol Cell Biol. 2003;4(6):446-456.

23. Rikitake Y, et al. Decreased perivascular fibrosis but not cardiac hypertrophy in ROCK1 $1^{+/}$haploinsufficient mice. Circulation. 2005;112(19):2959-2965.

24. Zhang YM, et al. Targeted deletion of ROCK1 protects the heart against pressure overload by inhibiting reactive fibrosis. FASEB J. 2006;20(7):916-925.

25. Liao JK, Seto M, Noma K. Rho kinase (ROCK) inhibitors. J Cardiovasc Pharmacol. 2007;50(1):17-24.

26. Shimizu Y, et al. Contribution of small GTPase Rho and its target protein rock in a murine model of lung fibrosis. Am J Respir Crit Care Med. 2001;163(1):210-217.

27. Shibuya M, et al. Effect of AT877 on cerebral vasospasm after aneurysmal subarachnoid hemorrhage. Results of a prospective placebo-controlled double-blind trial. J Neurosurg. 1992;76(4):571-577.

28. Breitenlechner C, et al. Protein kinase A in complex with Rho-kinase inhibitors Y-27632, Fasudil, and H-1152P: structural basis of selectivity. Structure. 2003;11(12):1595-1607.

29. Yamaguchi H, Kasa M, Amano M, Kaibuchi K, Hakoshima T. Molecular mechanism for the regulation of rho-kinase by dimerization and its inhibition by fasudil. Structure. 2006;14(3):589-600.

30. Thannickal VJ, Horowitz JC. Evolving concepts of apoptosis in idiopathic pulmonary fibrosis. Proc Am Thorac Soc. 2006;3(4):350-356.

31. Liu X, Kim CN, Yang J, Jemmerson R, Wang $\mathrm{X}$. Induction of apoptotic program in cell-free extracts: requirement for dATP and cytochrome c. Cell. 1996;86(1):147-157.

32. Yang J, et al. Prevention of apoptosis by $\mathrm{Bcl}$ 2: release of cytochrome $\mathrm{c}$ from mitochondria blocked. Science. 1997;275(5303):1129-1132.

33. Morita T, Mayanagi T, Sobue K. Dual roles of myocardin-related transcription factors in epithelial mesenchymal transition via slug induction and actin remodeling. J Cell Biol. 2007;179(5):1027-1042.

34. Small EM, et al. Regulation of PI3-kinase/Akt signaling by muscle-enriched microRNA-486. Proc Natl Acad Sci U S A. 2010;107(9):4218-4223.

35. Small EM, et al. Myocardin-related transcription factor-a controls myofibroblast activation and fibrosis in response to myocardial infarction. Circ Res. 2010;107(2):294-304.

36. Masszi A, et al. Fate-determining mechanisms in epithelial-myofibroblast transition: major inhibitory role for Smad3. J Cell Biol. 2010;188(3):383-399.

37. Crider BJ, Risinger GM Jr, Haaksma CJ, Howard EW, Tomasek JJ. Myocardin-related transcription factors $A$ and $B$ are key regulators of TGF- $\beta 1$ induced fibroblast to myofibroblast differentiation. J Invest Dermatol. 2011;131(12):2378-2385.

38. Miralles F, Posern G, Zaromytidou AI, Treisman R. Actin dynamics control SRF activity by regulation of its coactivator MAL. Cell. 2003;113(3):329-342.

39. Thomas SG, Huang S, Li S, Staiger CJ, Franklin-
Tong VE. Actin depolymerization is sufficient to induce programmed cell death in self-incompatible pollen. J Cell Biol. 2006;174(2):221-229.

40. Evelyn CR, et al. CCG-1423: a small-molecule inhibitor of RhoA transcriptional signaling. $\mathrm{Mol}$ Cancer Ther. 2007;6(8):2249-2260.

41. Liu F, et al. Feedback amplification of fibrosis through matrix stiffening and COX-2 suppression. J Cell Biol. 2010;190(4):693-706.

42. Hara Y, et al. Rho and Rho-kinase activity in adipocytes contributes to a vicious cycle in obesity that may involve mechanical stretch. Sci Signal. 2011;4(157):ra3.

43. Kimura K, et al. Regulation of myosin phosphatase by Rho and Rho-associated kinase (Rho-kinase). Science. 1996;273(5272):245-248.

44. Mueller BK, Mack H, Teusch N. Rho kinase, a promising drug target for neurological disorders. Nat Rev Drug Discov. 2005;4(5):387-398.

45. Huang X, et al. Relaxin regulates myofibroblast contractility and protects against lung fibrosis. $\mathrm{Am}$ J Pathol. 2011;179(6):2751-2765.

46. Tsukita S, Yonemura S. Cortical actin organization: lessons from ERM (ezrin/radixin/moesin) proteins. J Biol Chem. 1999;274(49):34507-34510.

47. Hinz B, Phan SH, Thannickal VJ, Galli A, Bochaton-Piallat ML, Gabbiani G. The myofibroblast: one function, multiple origins. Am J Pathol. 2007;170(6):1807-1816.

48. Rock JR, et al. Multiple stromal populations contribute to pulmonary fibrosis without evidence for epithelial to mesenchymal transition. Proc Natl Acad Sci U S A. 2011;108(52):E1475-1483.

49. Chua BT, Volbracht C, Tan KO, Li R, Yu VC, Li P. Mitochondrial translocation of cofilin is an early step in apoptosis induction. Nat Cell Biol. 2003;5(12):1083-1089.

50. Gourlay CW, Ayscough KR. The actin cytoskeleton: a key regulator of apoptosis and ageing? Nat Rev Mol Cell Biol. 2005;6(7):583-589.

51. Franklin-Tong VE, Gourlay CW. A role for actin in regulating apoptosis/programmed cell death: evidence spanning yeast, plants and animals. Biochem J. 2008;413(3):389-404.

52. Li S, Chang S, Qi X, Richardson JA, Olson EN. Requirement of a myocardin-related transcription factor for development of mammary myoepithelial cells. Mol Cell Biol. 2006;26(15):5797-5808.

53. Sasazuki T, et al. Identification of a novel transcriptional activator, BSAC, by a functional cloning to inhibit tumor necrosis factor-induced cell death. J Biol Chem. 2002;277(32):28853-28860.

54. Paavilainen VO, Bertling E, Falck S, Lappalainen P. Regulation of cytoskeletal dynamics by actinmonomer-binding proteins. Trends Cell Biol. 2004;14(7):386-394

55. Pollard TD, Borisy GG. Cellular motility driven by assembly and disassembly of actin filaments. Cell. 2003;112(4):453-465.

56. Bernstein BW, Chen H, Boyle JA, Bamburg JR. Formation of actin-ADF/cofilin rods transiently retards decline of mitochondrial potential and ATP in stressed neurons. Am J Physiol Cell Physiol. 2006;291(5):C828-C839.

57. Maloney MT, Bamburg JR. Cofilin-mediated neu- 
rodegeneration in Alzheimer's disease and other amyloidopathies. Mol Neurobiol. 2007;35(1):21-44.

58. Grinnell F, Zhu M, Carlson MA, Abrams JM Release of mechanical tension triggers apoptosis of human fibroblasts in a model of regressing granulation tissue. Exp Cell Res. 1999;248(2):608-619.

59. Fluck J, Querfeld C, Cremer A, Niland S, Krieg T, Sollberg S. Normal human primary fibroblasts undergo apoptosis in three-dimensional contractile collagen gels. J Invest Dermatol. 1998;110(2):153-157.

60. Carlson MA, Longaker MT, Thompson JS. Granulation tissue regression induced by musculocutaneous advancement flap coverage. Surgery.
2002;131(3):332-337.

61. Pardo A, Selman M, Kaminski N. Approaching the degradome in idiopathic pulmonary fibrosis. Int $J$ Biochem Cell Biol. 2008;40(6-7):1141-1155.

62 . Phan $\mathrm{SH}$. The myofibroblast in pulmonary fibrosis. Chest. 2002;122(6 suppl):286S-289S.

63. Hinz B. Tissue stiffness, latent TGF-beta1 activation, and mechanical signal transduction: implications for the pathogenesis and treatment of fibrosis. Curr Rheumatol Rep. 2009;11(2):120-126.

64. Iwasaki K, Hayashi K, Fujioka T, Sobue K. Rho/ Rho-associated kinase signal regulates myogenic differentiation via myocardin-related transcription
factor-A/Smad-dependent transcription of the Id 3 gene. J Biol Chem. 2008;283(30):21230-21241.

65. Wang R, et al. Angiotensin II induces apoptosis in human and rat alveolar epithelial cells. Am J Physiol. 1999;276(5 pt 1):L885-L889.

66. Golan-Gerstl R, Wallach-Dayan SB, Amir G, Breuer R. Epithelial cell apoptosis by fas ligand-positive myofibroblasts in lung fibrosis. Am J Respir Cell Mol Biol. 2007;36(3):270-275.

67. Waghray M, et al. Hydrogen peroxide is a diffusible paracrine signal for the induction of epithelial cell death by activated myofibroblasts. FASEB J. 2005;19(7):854-856. 